Revue d'histoire de l'Amérique française

REVUE D.HISTOIRE DE L'AMÉRIQUE FRANÇAISE

\title{
À l'origine des Cercles de fermières : étude comparée Belgique-Québec
}

\section{Yolande Cohen et Pierre Van Den Dungen}

Volume 48, numéro 1, été 1994

URI : https://id.erudit.org/iderudit/305299ar

DOI : https://doi.org/10.7202/305299ar

Aller au sommaire du numéro

\section{Éditeur(s)}

Institut d'histoire de l'Amérique française

\section{ISSN}

0035-2357 (imprimé)

1492-1383 (numérique)

Découvrir la revue

\section{Citer cet article}

Cohen, Y. \& Van Den Dungen, P. (1994). À l'origine des Cercles de fermières : étude comparée Belgique-Québec. Revue d'histoire de l'Amérique française, 48(1), 29-56. https://doi.org/10.7202/305299ar
Résumé de l'article

La constitution des Cercles de fermières relève autant d'un projet simultané des agronomes et moralistes chrétiens que de la mobilisation internationale des femmes rurales en vue de se doter de moyens efficaces de contrer l'exode rural. Dans le monde catholique, l'initiative revient aux moralistes belges, particulièrement désireux d'endiguer l'essor du socialisme et des idées sociales laïques dans les campagnes, suivie de près par le mouvement québécois. Dans les deux cas, toutefois, l'impulsion fondamentale des Cercles reste celle qui est donnée à ces mouvements par les femmes elles-mêmes, expliquant en partie les différences dans leur histoire respective : les Belges périclitant rapidement, les Québécois prenant un essor considérable au cours des années qui suivent leur fondation en 1915.

Ce texte propose d'analyser les origines des Cercles de fermières en Belgique et au Québec. Il tente de cerner les changements dans les discours et les principes fondateurs des Cercles, en particulier ceux de l'Église et des agronomes, de mettre à jour leurs objectifs et la façon dont les membres en ont disposés, à travers une histoire comparée menée sur des sources inédites
Tous droits réservés (C Institut d'histoire de l'Amérique française, 1994
Ce document est protégé par la loi sur le droit d'auteur. L'utilisation des services d'Érudit (y compris la reproduction) est assujettie à sa politique d'utilisation que vous pouvez consulter en ligne.

https://apropos.erudit.org/fr/usagers/politique-dutilisation/ 


\title{
À L'ORIGINE DES CERCLES DE FERMIÈRES: ÉTUDE COMPARÉE BELGIQUE-QUÉBEC
}

\author{
YOLANDE COHEN \\ Département d'histoire \\ Université du Québec à Montréal \\ PIERRE VAN DEN DUNGEN \\ Département d'histoire \\ Université libre de Bruxelles
}

\section{RÉSUMÉ}

La constitution des Cercles de fermières relève autant d'un projet simultané des agronomes et moralistes chrétiens que de la mobilisation internationale des femmes rurales en vue de se doter de moyens efficaces de contrer l'exode rural. Dans le monde catholique, l'initiative revient aux moralistes belges, particulièrement désireux d'endiguer l'essor du socialisme et des idées sociales laïques dans les campagnes, suivie de près par le mouvement québécois. Dans les deux cas, toutefois, l'impulsion fondamentale des Cercles reste celle qui est donnée à ces mouvements par les femmes elles-mêmes, expliquant en partie les différences dans leur histoire respective: les Belges périclitant rapidement, les Québécois prenant un essor considérable au cours des années qui suivent leur fondation en 1915.

Ce texte propose d'analyser les origines des Cercles de fermières en Belgique et au Québec. Il tente de cerner les changements dans les discours et les principes fondateurs des Cercles, en particulier ceux de l'Église et des agronomes, de mettre à jour leurs objectifs et la façon dont les membres en ont disposés, à travers une histoire comparée menée sur des sources inédites.

\section{ABSTRACT}

The establishment of the Cercles de fermières resulted from the efforts of Catholic agronomists and moralists, as well as an international mobilisation of women opposed to rural depopulation. From the Catholic side, the initiative came primarily from Belgian moralists, who were particularly anxious to counter progressive and socialist ideas in the countryside, although the movement in Quebec followed this initiative closely. In both cases, nonetheless, women proved to be the driving force of the movement, which explains in part the differences in their respective histories: the Belgian movement declined rapidly, whereas its counterpart in Quebec became very important following its establishment in 1915. 
This article will analyse the origins of the Cercles de fermières in Belgium and in Quebec. Through a comparative history based on previously unexplored sources, it will explore changes in the discourses of the principle founders of the Cercles, particularly those of the church and the agronomists, exposing their objectives as well as the uses made of these objectives by the members.

\section{INTRODUCTION}

À la recherche de moyens efficaces pour contrer l'exode rural, agronomes et moralistes chrétiens s'engagent dès la fin du XIX ${ }^{\mathrm{e}}$ siècle à donner aux jeunes filles le goût de l'air pur et de la bonne vie dans les campagnes. Une propagande concertée est alors mise en place, qui présente les femmes comme devant œuvrer prioritairement à l'assainissement et à la modernisation du monde rural. La science et la foi sont mobilisées dans un discours enthousiaste vantant les mérites de la vie rurale et de ses innombrables bienfaits. Bien que les objectifs des uns et des autres soient largement contradictoires, il apparaît très vite aux agronomes qu'ils ne pourront procéder à la réalisation de leurs objectifs de modernisation des campagnes sans l'assentiment des intéressées elles-mêmes, mais surtout sans l'appui du clergé.

Au même moment, de part et d'autre de l'Atlantique on assiste à l'émergence d'un mouvement de femmes rurales de développement certes inégal, selon les régions, mais qui frappe par son envergure. La concommitance de cet intérêt particulier pour les femmes rurales dans de nombreux pays occidentaux aboutit ainsi à la création d'un mouvement international des femmes rurales qui tient ses premiers congrès au début du siècle (1911 et 1912 aux États-Unis et 1913 à Gand en Belgique). C'est à ce dernier congrès que se définissent les orientations du mouvement, et que le «leadership» de la Belgique se précise en Europe. Les déléguées d'Italie, de Pologne, de Hongrie, de France, d'Espagne, etc., ne sont présentes au Congrès international de Gand en 1913 qu'à titre d'observatrices. Les cercles belges sont les véritables pionniers modèles de ce mouvement en Europe, lui insufflant à la fois des idéaux et proposant en exemple quelques troupes bien organisées. Le Québec lui emboîte le pas pour devenir avec le Canada anglais et les Etats-Unis les principaux pays et membres actifs de cette Internationale des fermières.

On a cherché à comprendre dans les discours originaux des moralistes chrétiens belges, et dans ceux des agronomes qui ont présidé à la fondation des Cercles de fermières du Québec, ce qui concourait à favoriser l'éclosion d'un tel mouvement. C'est à partir plus précisément de l'analyse des influences respectives du clergé, des agronomes et de l'État que nous avons cherché à identifier la création de cercles, 
dont l'existence et le déploiement sont spécifiques à chaque région de la Belgique et du Québec.

$\mathrm{Au}$ Québec, la polémique à ce sujet est vive, puisque dans les quelques études consacrées aux cercles (l'essentiel de la littérature sur le monde rural en ignore tout), on en attribue l'origine soit directement à l'Église ${ }^{1}$, soit à l'État ${ }^{2}$. Bien que l'ouvrage de Y. Cohen ${ }^{3}$ ait tenté de montrer combien les Cercles de fermières du Québec ont su jouer entre ces deux instances au profit de l'expansion de leur association, il reste de nombreuses résistances à cette hypothèse. Ces résistances relèvent d'un courant théorique d'orientation matérialiste ou marxiste, présent dans de nombreuses études historiques et féministes. On ne conçoit pas, selon ces hypothèses, que des femmes aient pu développer des associations qui ne soient partiellement ou entièrement inféodées à l'Église, à l'État ou aux deux. Selon ces théories, le pouvoir étant uniquement du côté des institutions de domination, les femmes et leurs associations n'ont qu'une faible marge de manœuvre pour y résister: leur aliénation résulte le plus souvent de ces rapports de domination qui ne leur laissent que quelques espaces d'autonomie relative (qui deviennent très vite ghettoïsés et marginalisés) ${ }^{4}$.

Il nous a semblé important de nuancer et même de remettre en question ce paradigme ${ }^{5}$. On a cherché à questionner le postulat d'une volonté de domination systématique de l'Église et de l'Etat à l'endroit des associations de femmes rurales. Une telle démarche a pour effet de montrer que courtiser des groupes suppose une altération des discours et des représentations que l'on en fait; par exemple, l'Église aura recours à la valorisation du métier de fermière et prônera les nouvelles techniques de la «science domestique» pour recruter des femmes rurales dans ses associations. Ces processus, loin d'être innocents, visent à l'intégration de couches nouvelles à la vie d'institutions comme l'Église ou l'État. Nous postulons donc que les femmes qui s'engagent dans le mouvement des femmes rurales prennent nettement conscience du nouveau rôle public qu'elles peuvent jouer, tant dans

1. Claire Chénard, Les Cercles de Fermières, une appellation tronquée, mémoire de maîtrise (sociologie), Université Laval, 1981, 217 p.

2. Jean Letendre, Les Cercles de fermières au Québec, 1915-1970, un exemple d'encadrement politique d'un mouvement populaire féminin, mémoire de maîtrise (histoire), Université de Sherbrooke, 1983.

3. Yolande Cohen, Femmes de parole. Histoire des Cercles de fermières du Québec, 1915-1990 (Montréal, Le Jour éditeur, 1990), 315 p.

4. Pour une discussion éclairée des tenants de cette orientation théorique et de quelques autres, voir Ruth Bloch, «A Culturalist Critique of Trends in Feminist Theory», CONTENTION, 2,3 (printemps 1993).

5. Avec quelques chercheurs, nous avons amorcé ce débat. Voir le numéro spécial «Le monde rural" de la revue Recherches sociographiques, 2-3 (1988). 
leurs associations qu'au plan plus global des stratégies d'intervention dans la sphère publique.

En Belgique, l'existence et l'importance de ces cercles n'a pratiquement pas retenu l'attention. Il n'existe à leur sujet aucun travail de synthèse. Ce désintérêt - y compris dans les travaux qui traitent de la «structuration» des campagnes sous la houlette du Boerenbond, le syndicat des fermières belges à dominante linguistique flamande (1891) - résulte très probablement de deux facteurs: d'abord, la polarisation du regard historique sur le monde ouvrier et sur le développement industriel, puis le peu d'avancement des études historiques sur les femmes. On ne peut guère mentionner, en effet, que quelques études sur les paysannes, centrées en général sur la période 1918$1940^{6}$.

C'est à partir de l'étude des origines des Cercles de fermières au Québec et en Belgique que nous avons choisi d'illustrer les éléments marquants de cette transition. On a aussi voulu comprendre comment le processus de création des cercles de fermières s'est déroulé, et pour quelles raisons les cercles n'ont pas connu en Belgique l'essor de leurs homologues du Québec. Au delà des raisons circonstancielles, qui font de chaque histoire nationale un cas particulier, nous avons voulu cerner les différences dans les discours et les principes fondateurs des cercles, leurs objectifs, et comment leurs membres en ont disposé. Bien qu'il soit difficile d'établir les liens qui existent entre des recommandations de principes et leur application, une des manières de les apprécier consiste à en faire l'histoire détaillée.

L'analyse comparative sert ici à recentrer le débat sur les rôles respectifs de l'Église et des agronomes dans la création des cercles. Il a fallu tenir compte du peu de sources disponibles en Belgique, puisqu'à notre connaissance il n'existe pas de travaux consacrés aux cercles. C'est grâce à Éliane Gubin, professeur à l'Université Libre de Bruxelles, que nous avons pu avoir accès aux sources et que nous sommes à même de tester les hypothèses avancées et largement vérifiées sur les cercles du Québec ${ }^{7}$.

6. H. Herrier, «L'Action catholique rurale féminine et son histoire», Cahier de l'Action catholique rurale des femmes, 13 (1990); P. Liebens, «Van Boerin tot eigen aard. Historick van de Boerinnenbond K.V.L.V.», Stappels, 7,2 (1988): 68-72; 7,3 (1988): 116-121; 7,4 (1988): 151158; C. Mougenot, «Un siècle d'évolution des femmes en milieu rural», Cahiers de l'Action catholique rurale des femmes, 9 (1989).

7. C'est sur sa recommandation également que Pierre Van Den Dungen s'est vu octroyé une bourse du Canada et qu'il a pu réaliser les dépouillements originaux que nous présentons dans la première partie de cet article. Qu'elle trouve ici l'expression de nos sincères remerciements. 


\section{I - LE CAS BELGE}

«Un cercle c'est une association de fermières et de ménagères rurales qui par des conférences, des bibliothèques, des concours, cherchent à répandre l'instruction professionnelle de la femme à la campagne $^{8}$.» Ces termes de l'abbé Polet, un des premiers fondateurs des «Cercles de Fermières» en Belgique, définissaient en 1911 les objectifs de ce qu'il concevait comme un nouveau type d'association féminine rurale. Il ne s'agit pas d'une initiative isolée et encore moins d'un projet expérimental: la création des cercles de fermières résulte d'une réflexion profonde et relève d'une idéologie connue.

Ces associations représentent dans l'esprit de nombreuses personnalités catholiques la solution - pratique et traditionnelle - pour endiguer l'exode rural. C'est, en effet, en assurant la stabilité et l'harmonie du ménage paysan, la bonne éducation des enfants, dans le respect des traditions, que les femmes rurales donneront à leur descendance l'envie de demeurer à la campagne.

Notons pourtant la modernisation du discours traditionnel de ces moralistes. Il faut instruire davantage les fermières pour qu'elles accomplissent encore mieux leurs tâches professionnelles et ménagères, mais aussi pour qu'elles prennent conscience de l'importance de leur rôle social à la campagne. Les femmes seront-elles plus sensibles à un discours qui les valorise non plus seulement comme mères et épouses, mais aussi comme fermières indispensables à la survie d'un mode de vie dont elles seraient les premières à bénéficier? Voyons dans quelles conditions les discours à l'endroit des femmes vont changer.

\section{A. L'influence des agronomes dans la définition du rôle des fermières}

La volonté d'instruire et d'organiser les fermières et les ménagères rurales est antérieure à la création des premiers cercles de fermières, nés en Belgique en 1906. Dès 1881, en effet, Alphonse Proost ${ }^{9}$, alors inspecteur général de l'agriculture, avait proposé une

8. Abbé Polet, Comment organiser des Cercles de Fermières? (Leuze, 1911), 8 p.

9. Fils de Prosper Proost, fondateur du Journal de Bruxelles, Alphonse Proost est né à Bruxelles en février 1847 et mort à Uccle en 1931. Il fait des études de sciences naturelles à l'Université Libre de Bruxelles. Mais comme il est un fervent croyant, il préfêre enseigner à Louvain où il occupe, à partir de 1879 , la chaire de Sciences naturelles appliquées à l'exploitation agricole à l'École d'agriculture de l'Université catholique de Louvain. Il est nommé secrétaire de la Société centrale d'agriculture en 1873. Il crée la Société scientifique de Bruxelles dans laquelle il propage ses théories favorables à l'agriculture scientifique. Eug. De Seyn, Dictionnaire biographique des sciences, des arts et des lettres, 2: 839. M. Rouvroy, Alphonse Proost (Bruxelles, Société belge d'éditions, coll. «Lettres et Arts belges», $\mathrm{n}^{\circ}$ 15, 1911), $112 \mathrm{p}$. 
vaste réforme éducative du secteur primaire. Un an plus tard, il part pour la Bretagne afin d'observer la première école agricole de «laiterie rationnelle» pour femmes qui préconise les plus récentes techniques dans le domaine et leur application dans la ferme. En 1883, il envoie à Coëtlogen, en Bretagne, une Flamande et une Wallonne qui, dès leur retour au pays, initient des classes ménagères, des syndicats laitiers et beurriers aux techniques locales.

Proost ne s'en tient pas là, puisqu'il encourage une politique de création d'écoles ménagères dans chaque région de Belgique. Dans la foulée, en 1896, il publie une brochure ${ }^{10}$ dans laquelle il explique sa doctrine du culte de la forme physique chez la femme, qui doit la ramener aux vrais principes des lois naturelles, loin de l'éducation esthétique qui privilégie les arts jugés artificiels. Favorable aux sciences naturelles et à ses aspects pratiques, opposé à la musique et à l'histoire, Proost encourage chez la femme l'apprentissage dans la nature, et la gymnastique qui la fortifie pour le bien de la race. Enfin, il recommande la création d'asiles en pleine campagne pour les jeunes filles pauvres et les pupilles afin de les initier à la vie en plein air dans le cadre d'un enseignement marqué par la religion. Mais c'est à la fin des années quatre-vingt-dix qu'il songe à créer des syndicats pour fermières, qu'il appelle «cercles» en référence aux premières associations du genre fondées en Ontario à partir de 1897.

\section{L'instruction}

Dès cette époque, il semble, en effet, que le ministère de l'Agriculture belge ait eu en son sein des éléments très au fait de la situation nord-américaine. Ainsi, Paul De Vuyst ${ }^{11}$, ancien étudiant d'Alphonse Proost à l'École d'Agriculture de Louvain, avait effectué un premier voyage d'études aux États-Unis en 1893, suivi d'un autre au Canada en $1904^{12}$. C'est au cours de ce second périple qu'il rencontre à

10. Alphonse Proost, L'Éducation de la femme selon la science (Bruxelles, 1896), $52 \mathrm{p.}$ Paru également en juillet 1895 dans la Revue des questions scientifiques, 12: 324.

11. Paul De Vuyst est né en 1863 à Borsbeke en Flandre occidentale. Docteur en droit et ingénieur agricole, il est délégué du comité permanent de l'Institut International à Rome. Nommé en 1893 inspecteur adjoint de l'agriculture, il est chargé du contrôle des écoles d'agriculture et du corps des agronomes de l'État pour la partie flamande du pays. Promoteur de nombreuses mesures en faveur de l'agriculture, il est d'abord promu au grade d'inspecteur principal en 1905 et devient directeur général du ministère de l'Agriculture, Grand Officier de l'Ordre de la Couronne et Chevalier de l'Ordre de Léopold. Eug. De Seyn, Dictionnaire biographique... (Éditions de l'Avenir), 1: 382. La Belgique active, monographie des communes belges et biographie des personnalités (Bruxelles, 1931), 167. Manifestations en l'honneur de monsieur Paul De Vuyst, 7 août 1930 (Bruxelles, Impr. industrielle et financière, 1930), 32 p.

12. Paul De Vuyst, L'amélioration de la vie rurale (Bruxelles, Commission internationale de L'Éducation familiale, 1938), $16 \mathrm{p}$. 
Toronto le ministre de l'Agriculture de l'Ontario, M. James, auprès duquel il constate l'efficacité des premiers cercles ontariens. De retour en Belgique, il ne cesse d'encourager la création de semblables associations; son accession en 1905 au poste d'inspecteur principal de l'agriculture lui facilite la tâche. Comme son maître Alphonse Proost, De Vuyst porte une attention particulière à l'instruction de la fermière, véritable garante de la survie des campagnes.

Il cherche à la fois à convaincre l'opinion belge du bien-fondé de sa démarche et à montrer aux pays étrangers que la Belgique est à la pointe du progrès social en milieu rural ${ }^{13}$. C'est pourquoi il institue des Pavillons de la Fermière lors des nombreuses expositions internationales organisées à travers le pays avant la Première Guerre mondiale: en 1905 à Liège, en 1910 à Bruxelles et en 1913 à Gand où, à cette occasion, un véritable village idéal moderne sera bâti de toutes pièces $^{14}$.

Des 1910, De Vuyst octroie une place de choix aux cercles de fermières à l'intérieur de ces pavillons. Il publie également, en 1907, Le rôle social de la fermière ${ }^{15}$, un livre bien accueilli dans le monde catholique belge, y compris par l'aile «féministe ${ }^{16}$ », plusieurs fois réédité, couronné par l'Académie royale de Belgique et traduit en anglais ${ }^{17}$. De Vuyst y constate l'effet bénéfique pour les campagnes de l'éducation et de la conscientisation des fermières, notammemt par l'intermédiaire des cercles.

\section{La science agricole}

Ces réflexions d'agronomes étaient donc à l'origine d'une politique plutôt favorable à l'éducation des femmes rurales. Proost, et à sa suite De Vuyst, avaient donné une nouvelle orientation à la politique sociale du ministère de l'Agriculture grâce aux postes de direction qu'ils y occupaient. Ils étaient inspirés par des exemples étrangers, et

13. J. Giele et F. Graftiau, Le pavillon de la fermière (Louvain, 1910), 86 p. Exposition Universelle et Internationale de Bruxelles en 1910.

14. Selon J. Giele, La ferme démonstrative (Exposition de Bruxelles, 1935), 70 p., ce type d'exposition se perpétuera jusqu'au milieu de l'entre-deux-guerres. Les pavillons reproduisaient des fermes modèles équipées des dernières innovations techniques à l'usage de la porcherie, de l'étable, ou du logis du fermier. Des élèves des écoles ménagères prouvaient aux visiteurs de l'exposition l'efficacité de ces découvertes par des démonstrations.

15. P. De Vuyst, Le rôle social de la fermière, son éducation professionnelle, la réunion des fermières, leur organisation à l'étranger, renseignements pratiques (Bruxelles, 1911), 194 p. Première édition, 1907.

16. Voir l'article favorable de Louise Van den Plas, «Le rôle social de la fermière», Féminisme chrétien de Belgique (mars 1907): 4. $151 \mathrm{p}$.

17. P. De Vuyst, Women's Place in Rural Economy. A Study in Sociology (London), 
particulièrement par les «Women's Institutes» ontariens, mais ils apportaient aussi une importante contribution personnelle. Nous verrons plus loin dans cette étude, lors de l'approche de l'expérience québécoise, combien le rôle des agronomes Désilets et Bouchard est comparable à l'action de Proost et De Vuyst.

Dans les deux cas, il s'agit d'hommes dotés d'une forte personnalité, convaincus de la nécessité d'une action sociale concertée à l'égard des femmes rurales et qui, grâce à leurs fonctions, ont pu initier un processus avec l'aide de l'État et mobiliser l'opinion publique dans le même sens. Ainsi, en 1903, la comtesse de Villermont, grande propriétaire terrienne, suit les conseils prodigués par le ministère de l'Agriculture lorsqu'elle met en place la première union professionnelle de fermières belges ${ }^{18}$.

Selon le département de l'agriculture, il revient, en effet, aux châtelaines et aux dames du monde d'encourager les familles à rester à la campagne et à propager la science agricole dans l'esprit du peuple ${ }^{19}$. Ce discours officiel sera maintenu jusqu'à la veille de la Première Guerre mondiale, puisque nous le retrouvons in extenso dans une déclaration faite par De Vuyst en 1913 lors de la réunion annuelle du Comité National de la Fédération des Cercles de Fermières ${ }^{20}$.

En outre, de nombreux périodiques agricoles - pour la plupart régionaux et proches du parti catholique au pouvoir - propagent à leur tour les thèses de l'éducation scientifique de la femme à la campagne. Pour une fois, une réforme relative aux femmes est accueillie sans réserve par les milieux catholiques; ses aspects, traditionnels en apparence, y sont évidemment pour beaucoup. Les différents commentateurs ${ }^{21}$ apprécient les aspects pratiques de la théorie, qui redéfinissent clairement les tâches des deux sexes à la campagne. Bref, la direction et la surveillance des travaux incombent à l'homme, alors

18. Voir le témoignage de De Vuyst, idem, et celui de M. D. Hallet qui considèrent le cercle de Villermont comme le premier de Belgique. «I: EEuvres féminines à établir à la campagne, II: Moyens d'assurer la vitalité des cercles», dans la première section du Congrès de fermières et de ménagères rurales, Namur, 13 décembre 1909, 36.

19. E. Loze, «La Belgique et ses femmes», Le repos dominical, bimensuel littéraire envoyé régulièrement dans tous les châteaux de Belgique, 35000 exemplaires, 17 (1905).

20. «Compte rendu du Comité national de la Fédération des Cercles de Fermières», Féminisme chrétien, 6: 3-4.

21. Lire notamment «L'Éducation des femmes à la campagne», L'Écho Wallon, 43 ( $1^{\text {er }}$ février 1913): 1; R. Delys, «La femme aux champs", Chasse et pêche, 7 (15 novembre 1908): 1-25; Zan P.I., «La femme en agriculture», Belgique horticole et agricole, 16 (15 août 1903): 245-246; Perbal-Rion, «La femme en agriculture», Le Luxembourgeois, 12 (1906): 161 162; et A. Schmitz, «La femme en agriculture», Union, 50 (10 décembre 1905); Le Luxembourgeois est le journal de la société agricole de la province de Luxembourg et l'Union, organe des associations chrétiennes du Luxembourg belge. 
que la femme déploie son activité à l'intérieur de la ferme, notamment dans son ménage, la laiterie et la basse-cour.

Il est question d'une véritable technicisation du travail et du savoir des fermières, aussi certains n'hésitent-ils pas à lui rappeler l'ensemble des devoirs qui occupent chaque instant de sa journée ${ }^{22}$. Pour le bien de son ménage, la fermière devra être première levée à l'aube et couchée la dernière, contente d'avoir rempli son rôle. Ouvrière agricole, métayère, journalière ou cultivatrice propriétaire, toutes ont des attributions et des pouvoirs différents en vertu de leur positon sociale, mais chacune travaille pour le bien de la nation.

Les journalistes martèlent ces recommandations dans un langage didactique et tentent de convaincre les jeunes filles, que le travail agricole rebute, par des dictons populaires comme: «le travail c'est la santé», «l'oisiveté est la mère de tous les vices».

\section{B. La récupération des cercles par les organisations catholiques}

Le premier cercle belge naît en décembre 1906 à Alveringhem dans la localité de Furnes-Ambacht en Flandre ${ }^{23}$; dès 1909, il compte déjà 220 membres $^{24}$. À travers le pays près de 60 autres cercles ont vu le jour, montrant à l'évidence, outre le dynamisme de leurs créateurs, un véritable engouement des femmes pour ce type de regroupement en milieu rural.

Initialement, le mode de fonctionnement des cercles varie selon les groupes, mais il se dégage néanmoins des constantes, comme la convocation des membres à quelques réunions annuelles au cours desquelles s'expriment des conférenciers, agronomes ou instructrices d'écoles ménagères agricoles. Les créations de cercles se décident souvent au sein des écoles ménagères agricoles, elles-mêmes sections d'écoles catholiques rurales.

Ainsi, à Leuze, localité de la province du Hainaut et siège en 1907 du premier cercle de la partie francophone du pays, l'école catholique, à l'initiative de son directeur le chanoine Moulart, prend une part importante dans l'élaboration des structures de cette première

22. R. Delys écrivait en substance: dès l'aube, fait le tour de son domaine; ramasse les œufs dans le poulailler; prépare le déjeûner; débarbouille les enfants; habille l'homme et la famille; donne le grain aux poules; visite la porcherie, les étables et l'écurie, prépare le repas de midi, veille à ce que personne ne profère de grossièretés à table, fait la vaisselle, range la cuisine et fait le linge, consacre son temps libre aux travaux de couture. "La femme aux champs», Chasse et pêche, 7 (15 novembre 1908): 125.

23. Louise d'Hondt (Wachtebeke), «Les Cercles de Fermières», Congrès des Cercles de Fermières et de ménagères rurales, Namur, 13 décembre 1909, 20-29, Rapports, Compte-rendu, Namur-Servais, 1909, 93-102-89-88 p., première section.

24. Idem, 22. 
association $^{25}$. Parfois, comme à Borsbeke en Flandre orientale, le cercle est directement affilié au groupe d'étude du personnel enseignant des écoles ménagères agricoles de la région ${ }^{26}$.

En somme, comme Proost l'avait souhaité, les associations de fermières constituent un complément à l'enseignement ménager agricole et permettent d'espérer, vu leur nombre croissant, une propagation rapide et durable du savoir "pratique» ou technique dans les campagnes. En revanche, la question du statut officiel des cercles reste ouverte, comme le constate M. D. Hallet, directeur de la Ligue Agricole Luxembourgeoise (masculine) à Arlon, au premier Congrès de fermières et ménagères rurales tenu en 1909 à Namur ${ }^{27}$. Les cercles pourraient cependant conserver, selon leur propre terminologie, le titre «d'association civile» financée par les cotisations des membres et par des subsides gouvernementaux.

\section{La question des femmes au Congrès catholique de Malines (1909)}

Il apparaît donc clairement qu'en Belgique, les premiers cercles sont issus des milieux agricoles catholiques, qu'ils sont animés par des catholiques et subventionnés par un gouvernement catholique... C'est pourquoi une place leur est faite lors du grand Congrès catholique de Malines qui a lieu du 23 au 26 septembre $1909^{28}$. Celui-ci comporte sept sections dont l'une est consacrée aux problèmes spécifiques des femmes dans le monde contemporain et aux solutions que, souvent, elles sont les seules à pouvoir apporter.

Cette section féminine se divise en trois groupes de réflexion consacrés, dans l'ordre, aux œuvres religieuses, aux œuvres charitables et, finalement, aux œuvres sociales et économiques parmi lesquelles figurent les Cercles de fermières. Signalons cependant qu'à la présidence des trois groupes siègent non pas des femmes mais des ecclésiastiques pour qui les rôles féminins s'inscrivent principalement dans une conception traditionnelle basée sur l'amour de la religion et la charité.

25. Mademoiselle la comtesse Pontbriand, présidente du cercle de fermières et de ménagères de Celles et des environs, "Les Cercles de Fermières dans le Hainaut, fondation et développement», Rapports présentés à la lère section du IIIème Congrès international des Cercles de Fermières de Gand, "les Associations de Fermières», Bruxelles, 1913, Comité national des Fédérations de Cercles de Fermières.

26. Madame Haentjens-Deleu, «Les Cercles de Fermières», Section I: 81-93; Congrès des Cercles de Fermières et de Ménagères Rurales, Namur, 13 décembre 1909.

27. Cf. l'intervention de Hallet au Congrès des Cercles de Fermières et de Ménagères rurales, Namur, 13 décembre 1909.

28. Congrès Catholique de Malines, 23-26 septembre 1909 (Bruxelles, Goemare, 1909), rapports, 2 volumes, 7 sections, section féminine: 211 p. CEuvres religieuses, charitables, sociales et économiques. 
L'action de la femme dans l'enseignement du catéchisme, en tant qu'auxiliaire de l'Église, est la priorité par excellence aux yeux des membres du congrès, et cela, malgré le nouvel idéal catholique social légitimé par l'encyclique Rerum Novarum de Léon XIII (1891), toujours en application sous son successeur Pie X. Dans les grandes lignes, il s'agit pour les laïcs, et surtout pour les femmes, de participer à la croisade de rechristianisation de la société en substituant à l'œuvre de bienfaisance, jugée à présent insuffisante, une action sociale plus appropriée aux nouvelles réalités quotidiennes ${ }^{29}$.

Ce combat s'impose pour beaucoup comme la réponse efficace aux tentatives de séduction des femmes catholiques par la gauche, mais aussi comme un retour aux préceptes de l'Évangile. Manifestement, le débat n'est plus d'ordre philanthropique mais politique et, une fois de plus, les femmes sont invitées à y prendre la place stratégique - qui leur revient. La charité, cette vertu théologale et féminine, se transforme en vertu militante. Les syndicats et sociétés d'études ont pour mission essentielle - du moins en Belgique - de récupérer les fidèles égarés dans les rangs socialistes.

Les cercles ne sont pas, en fait, l'outil essentiel de ce repositionnement stratégique, qui vise surtout le monde ouvrier et qui se concrétise par la création de syndicats féminins, sous la direction liégeoise de Victoire Cappe, soutenue par le cardinal Mercier ${ }^{30}$. Même s'il y est aussi question d'action sociale, les cercles contribuaient plus au maintien de traditions qu'à l'élaboration de réformes socio-économiques. C'est d'ailleurs comme tels qu'ils sont présentés dans le rapport rédigé par la baronne Rotsart de Hertaing à l'occasion du congrès.

\section{Le premier Congrès des Cercles de fermières en 1909}

Moins de trois mois après le Congrès de Malines, le 13 décembre 1909 , tout un congrès est consacré aux cercles à Namur, sous le haut patronage du directeur général de l'agriculture, Alphonse Proost ${ }^{31}$, de

29. Sylvie Fayet-Scribe, Associations féminines et catholicisme: de la charité à l'action sociale XIXe-XXe siècle (Paris, Éditions Ouvrières, 1990), 202 p., cite une déclaration de Pie X faite à l'évêque de Périgueux en 1904: «Dites-leur (aux femmes) bien que le Pape leur recommande l'action sociale. Il ne suffit plus qu'elles s'enferment dans les ouvres de bienfaisance proprement dites, où toujours l'on sent l'écart des rangs, la hauteur de celui qui donne et l'infériorité de celui qui recoit... Non, je leur demande d'aller au peuple, de lui rendre service dans une vraie confraternité chrétienne, suivant l'esprit évangélique lui-même.», 33.

30. V. Cappe, La Femme belge, éducation et action sociale (Louvain, Bibliothèque de la Revue sociale-catholique, 1912), 326 p., ainsi que son journal La femme belge, fondé en 1913, revue mensuelle traitant des questions morales, sociales, littéraires et artistiques.

31. A. Proost, «L'instruction et l'avenir de la femme à la campagne», communication à la lère section du Congrès de Namur, 1909, 58-73. 
l'évêque de Namur, $\mathrm{M}^{\mathrm{gr}}$ Heylen, et du gouverneur de la province, le baron Montpellier. La noblesse terrienne occupe quasiment toutes les places du comité d'honneur ainsi que celles du comité organisateur, secrétaires et président(e)s compris(e)s. Comme le souligne $\mathrm{M}^{\mathrm{gr}}$ Heylen lors de son discours de clôture, cet encadrement n'est pas sans en agacer certains:

Quelqu'un m'a dit, mais «il n'y a que des comtesses, des marquises, des baronnes, des femmes de grands propriétaires». Eh bien! s'il en est ainsi dans le comité n'en sera-t-il pas de même dans la direction des cercles de fermières; et alors comment les fermières auront-elles la liberté de discuter leurs intérêts en présence de leurs châtelaines, de leurs propriétaires, dont, ajoutait-on, les intérêts sont si souvent opposés à ceux des fermières ${ }^{32}$.

La critique n'est pas sans fondement, mais $\mathrm{M}^{\mathrm{gr}}$ Heylen l'élude en affirmant que, de toutes façons, fermières et châtelaines œuvrent pour la même cause sans se gêner mutuellement. Cette déclaration enchante la présidente Rotsart de Hertaing, elle-même de la noblesse, et disposée comme d'autres femmes de son rang «à aider le peuple et les cultivateurs ${ }^{33}$ ». En fait, le congrès, ouvert par une messe célébrée en présence de l'Évêque, était en de très bonnes mains. Au programme, des intervenants ingénieurs agricoles, des institutrices et des enseignantes d'écoles ménagères agricoles définissent les thèmes des conférences destinées aux cercles.

L'économie domestique, l'hygiène, et particulièrement l'hygiène alimentaire chez le cultivateur, ou encore des notions sur la gestion moderne des basses-cours et des jardins potagers constituent à leur avis les fondements de tout apprentissage pratique en milieu rural. Il faut, en tout cas, enseigner ces matières dans un esprit chrétien et affirmer le goût de la vie à la campagne chez la fermière ${ }^{34}$.

Les congressistes décident également du nombre idéal de conférences - entre quatre et six - à donner chaque année dans chaque cercle afin de ne pas gêner les occupations ménagères et professionnelles de la fermière. Ils réfléchissent donc aux meilleures façons de développer les cercles et ce, à la lumière des résultats acquis sur le terrain depuis 1907, mais aussi au moyen de vœux émis à la fin des interventions, à l'adresse des gouvernants, des président(e)s de cercles

32. Discours de $\mathrm{M}^{\mathrm{gr}}$ Heylen, Révérendissime Évêque de Namur, 4: 84-87, Congrès de Namur, 1909.

33. Discussion des Rapports, idem, 85.

34. I. Giele, «Les industries familiales comme moyen d'enrayer l'exode rural» dans la deuxième section du Congrès de Namur, 1909, 91-96. 
ou encore des fermières elles-mêmes. La référence hors frontières demeure les Women's Institutes de l'Ontario, forts à l'époque de plus de 12000 membres, comme le rapporte l'ingénieur agronome P. Van Biervliet dans sa communication ${ }^{35}$.

\section{La scission}

La création des Cercles de fermières ne faisait pas l'unanimité au sein du monde catholique. En effet, le chanoine Luytgaerens, alors secrétaire général du puissant Boerenbond, syndicat catholique des fermiers belges, ne peut accepter une association féminine qui, le succès venant, risquait de devenir autonome. Pour le Boerenbond, la perte de son influence sur les femmes signifie aussi la perte de son contrôle sur une part importante du monde rural. Aussi, dès 1907, crée-t-il une guilde à Terbanck près de Louvain, première d'une série qui en comptera près de soixante-dix, essentiellement en Flandre occidentale, en 1970.

Luytgaerens rédige la même année des statuts calqués sur ceux des Cercles de fermières et, en juillet 1911, constitue un «Boerinnenbond», c'est-à-dire un syndicat féminin mais placé sous la tutelle du Boerenbond, donc sous leadership masculin ${ }^{36}$. Notons cependant qu'il n'y a pas de conflit ouvert entre le Boerenbond et les cercles; au contraire, le curé de Terbanck est invité à y prendre la parole. Il s'agit plutôt d'une lente récupération des cercles, opérée par le syndicat des fermiers et réussie surtout en Flandre. La meilleure illustration de cette évolution réside dans le destin de De Boerin, le journal des Cercles de fermières flamands ${ }^{37}$, qui devient en 1911 Maandschrift van de Belgische Boerinnenbond, c'est-à-dire le mensuel des syndicats de fermières de Belgique, tandis que dans la partie francophone du pays, La Fermière, journal des cercles, reste entièrement sous le contrôle de l'association. Il est vrai que sa diffusion est confidentielle et limitée à la région de Tournai-Ath malgré les efforts de son fondateur, l'abbé Edgar Polet, unique rédacteur et secrétaire général ${ }^{38}$. Le périodique

35. P. Van Biervliet, «Les Cercles de Fermières de la Province d'Ontario, Canada», troisième section du Congrès de Namur, 1909, 34-39.

36. Leen Van Molle, Ieder voor allen, de Belgische Boerenbond 1890-1990 (KADOC, Leuven-Boerenbond belge, 1990), 392 p. (disponible en francais sous le titre Chacun pour tous, traduit du néerlandais par Étienne Cappelle); et Frans Sterckx, 100 jaar geleden, Uitgave van de Belgische Boerenbond (Leuven, 1991), 171 p. Non disponible en francais.

37. De Boerin, maandschrift voor de landelyke huishoudster Boerinnenkringen van de Vlaamsch Belgie, 1909-1914 (conservé au KADOC de Leuven-Louvain).

38. Abbé E. Polet, secrétaire général du journal La Fermière, à Leuze, «Utilité d'un journal pour fermières et moyens de lui donner de l'extension», dans la première section du Congrès de Namur, 1909, 31-34. Voir aussi sa brochure générale sur les cercles de fermières en note 1 . 
n'est d'ailleurs toujours pas reconnu comme organe officiel des cercles wallons en 1909, et Polet désire se décharger de sa tâche qu'il juge excessive.

Ainsi, à la veille de la Première Guerre mondiale, Luytgarens et son syndicat ont partiellement regagné du terrain, même si un nombre de cercles flamands, surtout en Flandre occidentale, restent affiliés à la Fédération nationale.

\section{Tableau 1}

Comité national des Fédérations de cercles de fermières ${ }^{39}$ sous la présidence de la baronne Rotsart de Hertaing

\section{Membres Dates}

A. Fédération des cercles du Boerinnenbond belge.

$15000 \quad 1914$

Pour la partie flamande du Brabant, les

fédérations provinciales anversoises,

limbourgeoises, de Flandres occidentale et orientale

B. Fédération des cercles du Brabant wallon

C. Fédération provinciale du Hainaut

D. Fédération des cercles de ménagères rurales de la province de Liège

E. Fédération luxembourgeoise des cercles

F. Fédération agricole de la province de Namur

Il apparaît clairement que le Boerenbond effectue un développement remarquable en peu d'années, grâce à son implantation dans la partie flamande du pays, à l'exception (considérable, il est vrai) de la Flandre orientale. Ainsi, les cercles, quoique implantés et regroupés en fédérations provinciales dans tout le pays, développent des structures différentes dans une bonne partie de la région flamande. En Brabant flamand, dans les provinces d'Anvers et du Limbourg et en Flandre occidentale (soit un total de 8902 membres en 1914), ils sont en effet eux-mêmes compris dans un large mouvement agricole masculin et bénéficient d'organes de presse largement diffusés. Ailleurs, c'est-à-dire en Flandre orientale et dans la partie francophone du pays - plus industrialisée, mais cela explique-t-il un nombre de

39. J. Giele ingénieur agricole, d'après les renseignements fournis par les bureaux des fédérations, Les Cercles de Fermières en 1914-1919 (Renaix, 1920), 39 p., Comité national des Cercles de Fermières sous le haut patronage de S.M. la Reine. 
membres moins important? - il existe un ensemble de fédérations placées sous la tutelle directe et exclusive du Comité national des Fédérations de Cercles de fermières.

\section{Le III Congrès international des Cercles de fermières (1913)}

Ces conflits d'influence, relatifs à la direction des cercles belges, n'empêchent pas le développement de la Fédération nationale ni sa reconnaissance par des groupements semblables qui se créent alors à l'étranger. L'organisation du $\mathrm{III}^{\mathrm{e}}$ Congrès international des Cercles de fermières à l'occasion de l'exposition universelle de Gand $^{40}$ est prise en charge par le Comité national des Fédérations.

C'est la première fois qu'une telle assemblée se tient dans un pays européen, les deux premières ayant eu lieu aux États-Unis en 1911 et en 1912. La présidente du comité organisateur est la baronne Rotsart de Hertaing, présidente du Comité national des Fédérations de Cercles belges, entourée de délégués venus des États-Unis, d'Amérique du Sud, de Grande-Bretagne, d'Autriche, de Hongrie, des Pays-Bas, de France, de Pologne et, bien sûr, du Canada anglais. Le congrès se penche sur les questions de laiterie, de potager, de basse-cour et d'éducation chrétienne de la fermière; et sur l'ensemble de ces sujets, on constate une remarquable unanimité chez les différentes délégations. Nous pourrions presque parler de la constitution d'une «internationale» en faveur de la lutte contre l'exode rural.

Parmi les délégués, seuls les porte-parole officiels sont présents. Remarquons l'admiration des représentants étrangers devant la rigueur des rapports d'activité produit par la délégation belge. Le travail présenté par l'agronome Giele est à cet égard un exemple du genre: très structuré, il propose cercle par cercle un résumé des activités et conférences organisées en $1912^{41}$. Il répertorie, en outre, les noms des conférencières, les titres de leurs conférences, le nombre d'auditeurs, le total des membres inscrits pour chaque cercle ainsi que les noms de leur président ou présidente et de leur secrétaire. D'autres interventions d'agronomes retiennent l'attention, telle celle du premier d'entre eux P. De Vuyst, alors directeur général au ministère de l'Agriculture

40. Voir «Les Associations de Fermières», Rapports présentés à la première section du III ${ }^{\text {eme }}$ Congrès international des Fermières de Gand, Bruxelles, 1913, Comité National des Fédérations de Cercles de Fermières; «Le rôle professionnel de la Fermière», Rapports présentés à la deuxième section du III ${ }^{\text {eme }}$ Congrès international des Fermières de Gand, Bruxelles, 1913, CNFF; et enfin Rapports $4^{\mathrm{e}}$ volume.

41. M. Giele, «L'activité des cercles de fermières en 1912», rapport présenté au nom du Comité national des Cercles de Fermières en Belgique, 9-94, dans la $1^{\text {tre }}$ section, «Les associations de Fermières», Congrès International de 1913. 
et délégué du bureau du comité organisateur du congrès ${ }^{42}$. Mais cet extrait de son rapport est révélateur de ses désillusions et des tensions qui ont surgi au sein même des cercles: «De même que l'enseignement du soldat doit être donné par ceux qui sont rompus aux questions militaires, de même les écoles professionnelles agricoles doivent être sous la direction du ministère de l'Agriculture ${ }^{43}$.» Bref, De Vuyst profite de la tenue du congrès international pour porter en pleine lumière la question du rôle reconnu des agronomes dans l'enseignement agricole et dans les cercles de fermières.

En 1909 déjà, lors du congrès des Cercles de fermières et de ménagères rurales, le problème avait été soulevé en cours de séance. Les nombreux ecclésiastiques présents, y compris Luytgaerens du Boerenbond, souhaitaient que l'enseignement professionnel des fermières se fasse en dehors du concours des agronomes de l'État, ce qui avait provoqué l'indignation des ingénieurs présents ${ }^{44}$. L'allocution de De Vuyst montre que la question reste ouverte en 1913. Selon les agronomes, envisager un programme d'économie domestique empreint de notions scientifiques, sans leur concours, est une hérésie pédagogique. En revanche, ils laissent de bonne grâce aux maîtresses des écoles ménagères le soin d'organiser et de donner les leçons.

Mais on comprend la démarche des dirigeants des cercles, religieux, nobles et notables catholiques, puisque, pour eux, toute intervention directe de l'État, c'est-à-dire toute aide autre que financière, engendre un risque d'affaiblissement de leur pouvoir d'influence. Or, comme l'indique le comte de Villermont appuyé par l'évêque de Namur, «nous aimons beaucoup les agronomes mais il est certain que nous ne voulons pas laisser à des laïcs le soin de l'instruction de nos enfants ${ }^{45}$ ». Prompte à réagir aux tentatives d'intervention des agronomes sur ce terrain, l'Église réitère sa suprématie dans le domaine de l'éducation.

Bien que Proost et De Vuyst ont joué un rôle considérable en faveur des écoles ménagères et doivent être crédités du bilan largement positif qu'afficheront les Cercles de fermières à la veille de la Première Guerre mondiale, ils sont très vite relégués à une place subalterne par des ecclésiastiques soucieux de confirmer leur emprise sur l'organisation et les structures mises en place. Pour les deux

42. P. De Vuyst, «La vulgarisation de la science agricole», $3^{\mathrm{e}}$ section, 1-20. «La fermière, mère de famille et ménagère», Congrès International de 1913.

43. Idem, 9.

44. Congrès des cercles de fermières et de ménagères rurales, Namur, 13-12 1909, Namur-Servais, 1909, 93-102-84-89 p., section 4.

45. Idem, section 4, «Discussion des rapports», 8 . 
agronomes impliqués dans la fondation des cercles, la tenue de ce congrès signifie à la fois l'affaiblissement de leur influence, écartés qu'ils sont de l'organisation des cercles par les ecclésiastiques, et le début du rayonnement de leurs idées à l'étranger. En ce sens, l'aspect moderniste qu'ils préconisent pour les cercles et le développement d'une économie domestique plus rationnelle et scientifique trouveront un écho plus large à l'étranger qu'en Belgique.

C'est au Québec que le travail de l'agronome belge Paul De Vuyst a le plus retenu l'attention, notamment au sein du ministère de l'Agriculture, lui aussi préoccupé par le problème de l'exode rural ${ }^{46}$. Ses fonctionnaires (au premier rang desquels se trouve Alphonse Désilets) s'inspireront largement et des principes et des structures du mouvement belge. Ceci peut paraitre paradoxal vu la proximité géographique des Women's Institutes de l'Ontario. Mais les liens culturels et la proximité religieuse jouent un rôle plus grand que la proximité géographique. Tâchons de mettre à jour les principaux éléments d'une telle connivence entre les fondateurs des cercles belges et québécois, dont nous savons que l'usage de la langue française et la pratique de la religion catholique (élément fondamental dans la mesure où les membres des cercles en Flandre et même à Luytgaerens parlent flamand et/ou français) constituent les principaux liens.

\section{II - L'ASSOCIATION DES CERCLES DE FERMIÈRES DU QUÉBEC: ÉLÉMENTS DE COMPARAISON}

C'est en 1915 à Chicoutimi, puis à Roberval, que sont fondés les premiers cercles sous l'égide des agronomes Bouchard et Désilets ${ }^{47}$. Comparativement à la Belgique, le mouvement démarre au Québec quelque 10 ans plus tard et sera plus lent à s'étendre, puisque ce n'est qu'à partir de 1926 qu'il comptera une centaine de cercles et 6225 membres. Pourtant, bien que décalée dans le temps, la comparaison s'impose en raison de l'influence déterminante des organisateurs belges sur la constitution des cercles au Québec. Une telle comparaison devra certainement tenir compte des transformations majeures qui ont eu lieu dans le monde rural au sortir de la guerre; elle vise toutefois à mettre en lumière les questions plus spécifiques des influences

46. International Congress van Boerinnenbond - Congrès International des Cercles de Fermières, Augustus - 9-10-11-août 1930, Antwerpen, Anvers, 1930, 471 pages, où l'on trouve aux pages 352-357 la «carte de visite» des cercles québécois sous la forme de réponses données à un questionnaire envoyé par les organisateurs du Congrès. On lit que les cercles ont été fondés d'après les conseils de $M$. De Vuyst qui a toujours suivi de près leur développement et leur a donné de judicieux conseils.

47. Rapports du ministère de l'Agriculture de la province de Québec pour l'année 1915 (Québec, 1916). 
réciproques du mouvement du catholicisme social sur l'organisation des femmes rurales dans les deux pays considérés. Nous nous attarderons à développer les aspects comparatifs des cercles en utilisant certains résultats auxquels nous sommes parvenus à partir de la monographie qui fut consacrée à leur histoire ${ }^{48}$.

\section{A. Le rôle décisif des agronomes Désilets et Bouchard}

Professeur à l'École d'agriculture de Sainte-Anne-de-la-Pocatière, Georges Bouchard s'était rendu à la veille de 1914 en Belgique et avait constaté le dynamisme des cercles belges. De son côté, Désilets prône la création de regroupements semblables à ceux de la Belgique au congrès de la Jeunesse Catholique tenu en 1914. Associés de près aux activités des agronomes belges, Bouchard et Désilets s'engagent à fond dans la croisade en vue de constituer des cercles au Québec. Ils reprennent à leur compte la réthorique des agronomes belges sur la modernisation du monde rural par l'éducation scientifique des femmes et ils n'hésitent pas à se faire les promoteurs de cette vision tant auprès des femmes qu'auprès du ministère de l'Agriculture. En mettant leur programme au service de ce dernier, ils effectuent la même démarche que leurs confrères belges. Ils éviteront toutefois de laisser l'organisation sur le terrain entre les mains du clergé, mais se garderont bien de le heurter en inscrivant leur démarche dans l'action sociale cléricale. Dès les premières créations de cercles, ils constitueront une structure d'intégration des cercles au ministère de l'Agriculture.

Tout d'abord, Désilets prend la direction effective de l'organisation qu'il contribue à fonder peu de temps après sa déclaration en 1914. Soucieux de donner aux cercles une orientation uniforme, il règne en maître sur toutes les créations de cercles qui doivent obtenir son autorisation pour être affiliés à l'association, au moins jusqu'en 1929. Pour ce faire, il crée au sein du ministère de l'Agriculture une section d'économie domestique qu'il dirigera et de laquelle dépendront les cercles. Il édicte ensuite des règlements stricts concernant la création, le fonctionnement et les activités des cercles qui seront approuvés par le premier congrès général des cercles en 1922.

Ainsi, on s'oriente dès les débuts vers une organisation qui fait de la pratique de l'économie domestique une condition essentielle de l'adhésion des femmes aux cercles. Plus qu'un club de femmes rurales, le cercle serait l'équivalent d'une association professionnelle

48. Y. Cohen, Femmes de parole. L'histoire des Cercles de Fermières du Québec, 19151990 (Montréal, 1990), 315 p. À moins d'indications contraires, toutes les références subséquentes sont tirées de ce livre. 
de fermières. On y répond au besoin de moderniser l'agriculture par des techniques appropriées diffusées tant par les écoles ménagères que dans les cercles. Dotés d'une revue trimestrielle, La Bonne Fermière, que dirige la femme de Désilets, Rolande, les cercles apparaissent comme une courroie de transmission idéale des directives du ministère de l'Agriculture et de ses agronomes aux femmes rurales. En revanche, les cercles membres y trouvent aussi une valorisation de leurs activités domestiques et agricoles, ainsi qu'une reconnaissance officielle de leur production, qui se traduit par des subsides gouvernementaux aux activités de tissage, d'apiculture, etc. Soucieux également de ne pas heurter les susceptibilités locales, surtout celles du clergé, Désilets insiste pour subordonner la fondation des cercles à un comité de parrainage formé du curé de la paroisse et de l'agronome de la région ${ }^{49}$.

L'exemple du Cercle de Macamic dans le comté d'Abitibi-Ouest est à ce point de vue instructif. Douze femmes souhaitaient constituer un cercle après avoir assisté à un cycle de conférences données par des envoyés du ministère, mais elles s'étaient heurtées à l'hostilité de l'agronome du comté. Informé de la situation, Désilets s'empresse de soutenir l'agronome et le fait savoir aux intéressées par une lettre dans laquelle il les prévient «qu'il ne donnera suite qu'aux entreprises approuvées par M. Rioux [l'agronome]...» Il ajoute encore: «Il m'a déjà écrit au sujet de l'établissement de quelques Cercles de fermières dans ce district et je vous répète que nous ne pouvons ni ne voulons marcher en dehors de sa conduite officielle ${ }^{50} . »$

Malgré des mois de démarches auprès des autorités concernées, le groupe de femmes n'a pu parvenir à son but. Les archives des cercles ne révèlent cependant qu'un cas de ce genre, aussi serait-il excessif de généraliser, d'autant plus qu'à partir de 1925 les demandes de créations de cercles se multiplieront tellement que le ministère n'aura plus les moyens d'appliquer une politique aussi dirigiste. Toutefois, la question de l'orientation étatiste ou agronomiste des cercles du Québec à leurs origines demeure problématique, compte tenu de l'influence non négligeable de l'Église dans cette province. La comparaison avec l'influence qu'a eue l'Église sur les cercles en Belgique peut contribuer à faire comprendre celle du Québec.

49. S. Marchand et $\mathrm{Y}$. Cohen, «Les relations entre les cercles de fermières et l'État à travers leur correspondance (1920-1968)», communication présentée dans le cadre du colloque Le mouvement des fermières au Québec, ACFAS, mars 1989, 32 p.

50. Lettre de A. Désilets, 5 novembre 1920, à madame Ovide Pépin. ANQ, E009, 253. 


\section{B. Le rôle de l'Église}

Contrer l'exode rural, tel est l'objectif premier affirmé par les cercles belges et québécois. Certes, les arguments des uns et des autres varient selon que l'on tente de préserver les milieux ruraux du socialisme, comme en Belgique, ou que l'on cherche à renforcer l'identité canadienne-française que l'on croit menacée au Québec par l'urbanisation et les autres fléaux de la modernisation ${ }^{51}$. Et même si l'on trouve une semblable aversion envers l'urbanisation en Belgique, l'appel de l'Église au sentiment national y est moins problématique. Cette composante est propre au catholicisme du Québec et colore d'autant l'action et l'idéologie des cercles. Ce sont ces particularités qu'il faut appréhender plus précisément pour mieux comprendre les développements respectifs de ces associations. Indépendamment de la dynamique propre des cercles et du mouvement qu'ils impulsent, qui est l'objet principal de la monographie que leur consacre $Y$. Cohen et dont nous ne traiterons pas ici, ce qui est en cause, ce sont bien les applications particulières au Québec et à la Belgique de la doctrine sociale de l'Eglise à l'égard des femmes rurales. En somme, du fait de l'essor important du socialisme dans les milieux ouvriers, la question sociale est dès la fin du XIX $\mathrm{X}^{\mathrm{e}}$ siècle en Belgique comme dans le reste de l'Europe objet de préoccupations majeures.

\section{Le catholicisme social}

La charité chrétienne ne peut plus à elle seule constituer une politique pour l'Église. Constitués dans le but avoué de servir de base à la rechristianisation des populations touchées tant par le socialisme que par la modernisation, des groupes intermédiaires (associations, syndicats, mutuelles, etc.) prolifèrent dans les deux pays avec des résultats toutefois différents. Bien qu'elles soient représentées dans les discours comme un élément important de cette reconquête, les femmes rurales ne sont cependant pas investies du même intérêt de part et d'autre de l'Atlantique et ce, d'autant plus que la Grande Guerre modifiera durablement les choses.

Dès leur fondation, les cercles belges sont l'objet de la sollicitude de l'Église et du Parti Catholique qui représente ses intérêts et compte en ses rangs de nombreux ecclésiastiques, au point qu'il est difficile

51. Voir un exemple de ce type de discours tenu jusque dans les années soixante: Abbé Georges Bilodeau, Le vrai remède, étude de la crise actuelle, remèdes proposés (Québec, L'Action sociale, 1931). Il souligne l'apport vital des agriculteurs pionniers au Canada francais, ces «aïeux, sans lesquels le pays n'aurait eu (et n'aura) aucune chance de survivre face à l'américanisation symbolisée par les villes malsaines». 
de savoir à qui de la «famille catholique» ou des agronomes, souvent eux-mêmes issus des écoles catholiques, en reviendra la paternité. En 1907, les éléments conservateurs de l'Église belge s'appuient solidement sur les cercles pour propager leur message de solidarité entre les classes, de réveil chrétien et de valorisation du monde rural dont les fermières seraient les vecteurs. Ils investissent l'ensemble de l'organisation, en s'attribuant les postes de direction et en faisant des notables leurs principaux alliés. Il y est rapidement question de défense de la morale, chrétienne par essence. Le rôle social conservateur de la femme et la défense de la religion prennent le pas sur les aspects agricoles.

Au Québec, dix ans plus tard, l'Église est encore largement associée au développement initial des cercles; les membres du clergé n'occupent toutefois que des postes honorifiques dans l'organisation. Or, dans la mesure où l'association fonctionne sur des bases agronomiques et non ecclésiastiques, le fossé entre les deux ne tardera pas à se creuser aboutissant ultimement en 1940 à la rupture radicale avec l'Église. En somme, l'option imposée par Désilets à l'origine de l'association, à savoir une affiliation en bonne et due forme des cercles au service d'économie domestique du ministère de l'Agriculture est renouvelée volontairement quelque 15 ans plus tard par les cercles, quand ils décident de rejeter la proposition des évêques voulant leur affiliation aux districts diocésains. Comment une organisation de femmes catholiques comme les cercles échappe-t-elle presque entièrement au contrôle de l'Église?

La comparaison avec la Belgique apporte certains éclaircissements à cette question. Elle suscite d'abord une série d'interrogations: pourquoi le clergé canadien-français laisse-t-il l'organisation des cercles entre les mains des agronomes, alors que son homologue belge est si prompt à réagir et à presque les écarter? L'idée qu'une association de femmes rurales puisse un jour échapper au contrôle de l'Église était-elle à ce point impensable? Doit-on croire qu'il pouvait y avoir en ce domaine interchangeabilité ou répartition des rôles assumés par l'Église et l'État au Québec dans les années 1920? Au clergé reviendrait la charge de veiller à ce que les principes moraux de mariage, de reproduction, etc., soient strictement respectés par les cercles grâce à leurs aumôniers, tandis que Désilets et son service s'occuperaient de leurs besoins matériels.

En Belgique, une situation semblable aboutit à des résultats diamétralement opposés en ce qui concerne l'organisation des cercles. L'influence des agronomes s'arrête aux bonnes idées qu'ils diffusent et surtout à celle de valoriser le travail réalisé par les fermières. Nous croyons que c'est parce que certains ecclésiastiques sont largement 
acquis à la volonté d'organiser le monde rural, et en particulier les femmes; ils adaptent son discours et ses principes afin de pouvoir investir et s'emparer de l'organisation des cercles à son profit. Par un effet de mimétisme et parce qu'ils observent les progrès réalisés par. les tenants du catholicisme social dans le monde ouvrier, les éléments les plus conservateurs du clergé belge n'hésitent pas à faire de même dans le monde rural plus traditionnel.

En ce sens, l'étude des origines des cercles belges nous montre bien le cas d'une organisation contrôlée par le clergé. À deux reprises, en 1909 et 1913, l'Eglise met au pas les agronomes en rappelant ses prérogatives sur les contenus de programmes éducatifs destinés aux fermières... et obtient gain de cause. De même le chanoine Luytgaerens crée ses propres cercles pour se prémunir contre le risque de perdre le contrôle des femmes rurales flamandes.

Le décalage d'une dizaine d'années entre les dates de fondation des cercles belges et québécois joue certainement un rôle dans la laïcisation presque immédiate des cercles au Québec. Car au Québec en 1920, l'Église ne semble pas se soucier de l'organisation des femmes rurales, si ce n'est pour articuler un discours sur les bienfaits de la vie rurale et leur rôle dans le maintien des familles en milieu rural. Le champ de l'organisation étant libre, l'État, par ses agronomes, n'a pas de mal à répondre à leur demande et à se frayer un chemin qui deviendra un bastion, puisque les cercles seront une des premières associations de femmes laïques catholiques à voir le jour au Québec. Certes, il ne faut pas sous-estimer l'impact local de l'action du clergé qui soutient largement les pionnières de ce mouvement, ces élites féminines locales. Toutefois, cette absence relative d'initiative de la part de l'Église lors de la fondation des premiers cercles au Québec s'explique dans la mesure où il n'existe pas encore chez ses idéologues d'intérêt suffisant pour la question sociale. Ce n'est qu'à partir des années 1930 que se développe le courant de pensée incarné par la figure d'Esdras Minville ${ }^{52}$, disciple de l'abbé Groulx et théoricien inspiré par la doctrine du "corps intermédiaire». Minville déplore la part importante prise par l'État dans les associations et de façon générale dans les rapports sociaux entre citoyens, considérant qu'une telle immixtion porte atteinte à l'autonomie de la collectivité. Il envisage une réforme pour lutter contre cette situation et souhaite le rétablissement d'une activité économique à caractère rural, ce qui le rapproche sur ce point des agronomes.

52. R. Payette, Esdras Minville et le problème national canadien-français (1923-1956), mémoire de maîtrise (histoire), Université du Québec à Montréal, 1983, 323 p. 
On peut penser que ces réflexions ont conscientisé le clergé québécois, comme ce fut le cas des hommes d'église belges lorsqu'ils découvrirent les thèses des encycliques vaticanes leur faisant prendre conscience de la nécessité d'encadrer sérieusement les associations sur le terrain. Mais à l'époque, les Cercles de fermières avaient déjà acquis une autonomie relative: les agronomes eux-mêmes ne peuvent plus complètement les diriger. Aussi, la seule alternative laissée au clergé québécois sera de constituer un mouvement concurrent, ce qu'il fera en créant l'Union Catholique des Fermières (UCF) en 1945, avec le succès mitigé que toute scission entraîne. Minville et ses disciples groupés au sein de l'École Nationale désirent donc, à l'instar de nombreux ecclésiastiques belges et européens, offrir aux fidèles une réinterprétation de la doctrine sociale de l'Église à la lumière des exigences de l'époque moderne en apportant à leurs réflexions une dimension nationale particulière. L'idée de base du catholicisme social est cependant la même de part et d'autre de l'Atlantique: il s'agit, audelà des divisions de classes, de revenir à l'individu comme membre de l'indissoluble communauté des fidèles, sous la direction spirituelle et temporelle de l'Église.

Cette nouvelle doctrine s'oppose à l' «ultra-capitalisme», au libéralisme débridé, porteur d'un individualisme nuisible à l'ensemble de la communauté, et au socialisme qui distingue des classes aux intérêts contradictoires là où, selon le bon chrétien, il ne peut y avoir qu'un seul corps social. En d'autres termes, il s'agirait d'une troisième voie dans laquelle l'Église et le Parti Catholique prendraient la première place comme principaux intermédiaires entre les hommes et les femmes, et où l'agriculture, garante d'un mode de vie traditionnel et moral, redeviendrait la première industrie. À ce titre, les Cercles de fermières constituent une des réalisations de cette nouvelle vision du catholicisme social, très corporatiste, voulue par les plus hautes sphères ecclésiales dès la fin du XIX ${ }^{\mathrm{e}}$ siècle.

Dans la pratique, ces préceptes ne se réalisent que partiellement et à des moments différents. Au Québec, le clergé est loin d'être acquis. Dès les années vingt, une partie du clergé s'indignait de l'absence de la mention «catholique» dans les statuts et règlements des cercles ${ }^{53}$. Certains évêques estimaient même qu'il était de leur devoir de créer une association catholique féminine semblable aux cercles mais plus proche de l'Église, tel l'archevêque de Rimouski, Monseigneur Courchesne, qui écrivait que «sans trop aider, le curé fera bien d'organiser selon les exigences de l'esprit chrétien un comité paroissial d'action

53. Claire Chénard, Les Cercles de Fermières, une appellation tronquée, mémoire de maîtrise (sociologie), Université Laval, 1981, 217 p. 
catholique féminine ${ }^{54} \gg$. Toutefois il faut attendre les années trente pour qu'une coalition prenne forme. Les évêques des diocèses de Sherbrooke, de Joliette ou encore de Saint-Jean-de-Nicolet condamnent ouvertement l'action des cercles qui, portés par leur succès, deviennent des associations trop autonomes à leur goût. Ce sont les tendances les plus conservatrices du clergé québécois qui contestent aux cercles leur trop grande indépendance face à l'Église. Impuissants, seuls à imposer leur règle, ils seront relayés et appuyés par des groupes modérés et réformateurs, soucieux de chercher des relais à leur doctrine. Il faudra encore une dizaine d'années pour voir émerger un front uni du clergé contre les cercles. Après l'échec de nombreuses négociations avec les cercles, la scission est inévitable. L'Assemblée Épiscopale des Archevêques et des Évêques de la Province décide d'appuyer, le 4 décembre 1945, la fondation de l'UCF (l'Union catholique des fermières). On ne peut s'empêcher de comparer cette entreprise aux agissements du Boerenbond belge, lequel en agissant très tôt et très vite a partiellement réussi là où ses homologues québécois ont échoué après avoir laissé la situation se détériorer.

Les cercles demeurent, en effet, la première association féminine rurale du Québec et ce, malgré de nombreuses défections ${ }^{55}$, davantage provoquées par l'intimidation exercée par certains curés - qui allaient jusqu'à refuser la communion à des adhérentes restées fidèles aux cercles - que par conviction ${ }^{56}$. En fait, les agronomes eux-mêmes seront dépassés par l'engouement des fermières qui se traduit par une multiplication du nombre de cercles et par la persistance du mouvement à s'en tenir aux principes et à l'organisation adoptés en 1922.

On peut avancer l'hypothèse que la division des ecclésiastiques québécois face à l'attitude à prendre devant les cercles, a permis aux fermières de constituer une association dynamique, véritable groupe de pression auprès du ministère de l'Agriculture, et bientôt de l'État. Dégagés de l'obligation de se soumettre, comme les cercles belges, à la tutelle des organisations rurales masculines (par exemple de l'Union catholique des cultivateurs, pourtant très active), les cercles québécois

54. M. Dumais, «Perspectives pour les femmes telles que proposées par un évêque entre 1928-1950», Revue d'histoire du Bas-Saint-Laurent, 5,3-4 (déc. 1978), 35 p. L'auteur reprenait un extrait de la déclaration de $\mathbf{M}^{\mathrm{gr}}$ Courschesne dans Circulaire au clergé, 30 (11 février 1933), 270 p.

55. G. Desjardins, Les cercles de fermières et l'action féminine en milieu rural entre 1915 et 1944, mémoire de maîtrise, Université du Québec à Montréal, 1983, p. 113. Elle parle de 10000 défections, soit près du quart des membres à l'époque. Au bout de quelques années, l'association parvient même à récupérer une partie de ses membres.

56. Y. Rialland-Morissette, Le passé conjugué au présent, Cercles de fermières du Québec. Historique 1915-1980 (Montréal, Éditions Pénélope, 1980), 144. 
ont réussi à se constituer une enclave au sein de la structure administrative du ministère, qui leur a permis d'agir avec beaucoup d'autonomie. Les fermières belges, de leur côté, ont dû tenir compte des groupements masculins déjà engagés dans des conflits d'intérêts et dans des luttes idéologiques pour lesquelles l'appui des femmes était recherché. Devant l'irréductible opposition de certains évêques et en l'absence d'un mouvement d'action sociale catholique au Québec en 1915 , les cercles parviennent à éviter de placer leur organisation sous la tutelle de l'Église pendant plusieurs années.

\section{CONCLUSION}

Cette analyse centrée sur les influences respectives de quelques agronomes et de certains membres du clergé dans la fondation des Cercles de fermières en Belgique et au Québec ne prétend pas épuiser les nombreuses questions que cette histoire soulève. Conscients d'avoir ignoré des aspects importants du problème, tels le poids relatif du corps naissant des agronomes professionnels dans l'appareil d'État belge et québécois, le rôle spécifique de cet appareil et de ses gouvernants dans la mise sur pied d'un enseignement technique agricole par exemple, les objectifs poursuivis par les associations elles-mêmes, etc., nous avons voulu signaler quelques-unes des pistes qui pourraient être approfondies par une étude comparative. Nous avons tenté l'analyse des discours particuliers et leur incidence spécifique sur la vie d'une organisation comme les Cercles des fermières qui permet de souligner plusieurs éléments. Tout d'abord, les femmes comme les hommes ne sont pas indifférents aux discours qu'on leur adresse. Cela peut paraître un constat banal, mais il faut le dire, car les études matérialistes des groupes de femmes semblent vouloir ignorer leurs rapports avec les idéologies en affirmant qu'elles sont indistinctement opprimées par l'une ou l'autre, ce que nous retrouvons sous l'expression, fréquemment utilisée pour caractériser la condition des femmes au Québec, d'aliénation due à l'oppression «clérico-nationaliste». On observe donc au terme de cette étude une différence importante dans les statuts des cercles: selon qu'ils sont sous la tutelle de l'Église comme en Belgique, ou celle de l'État comme au Québec, ils seront plus ou moins capables de s'en affranchir. Ainsi on peut croire que leur inscription idéologique et organisationnelle aura pour effet de réduire la marge de manœuvre des cercles belges et aura une incidence sur leur évolution ultérieure, étroitement associés qu'ils sont à l'Église. D'autant plus qu'en Belgique, l'État est aux mains des catholiques qui gouvernent sans partage de 1884 à 1914 et mettent tout l'appareil administratif au service de l'Église. La montée du socialisme ajoute encore à cette hégémonie quasiment théocratique à 
cause de la peur «des rouges» qu'elle suscite dans les milieux conservateurs.

$\mathrm{Au}$ Québec, les liens, apparemment très rigides, qui se sont formés entre les cercles et le ministère de l'Agriculture ne sont pas hégémoniques par nature, du fait même de la différence qui existe entre le type de rapport que ces associations entretiennent avec l'Église et l'Etat. Avec l'Église, les liens sont de type organique; avec l'État les liens sont plutôt contractuels. Ainsi la création de l'École ménagère agricole de Roberval en 1882, modèle imité par les Belges dans les années 1890, a incontestablement servi de terreau aux Cercles de fermières. Certes, ces conditions qui déterminent la création des cercles ne perdurent pas et seront éventuellement remises en question, mais elles restent incontournables pendant une bonne génération, sinon plus.

Ensuite, il est important de noter combien les discours à l'endroit des fermières ont changé. Pour amener les femmes à s'engager dans les cercles, l'Église doit changer sa rhétorique et adopter un ton et un contenu nouveaux; ce qu'elle fait en Belgique, en empruntant aux agronomes certains thèmes de leur discours (l'instruction et le rôle social moderne des femmes par exemple). Au Québec, où ce sont les agronomes eux-mêmes qui les dirigent, les fermières préfèrent se regrouper dans un autre type d'organisation que celui, figé, offert par les évêques de Rimouski ou de Trois-Rivières. Plutôt que de voir, dans ces nuances et changements de discours, des effets qui ne visent qu'à mieux manipuler les femmes, ne pourrait-on pas dire que des transformations majeures sont en cours dans la vision que l'Église a des femmes et de leur rôle? En ce sens, ces changements d'appréciation sont effectivement survenus, et les femmes membres des cercles ont pu largement en bénéficier. S'il est complexe de savoir, à cette étape si les cercles ou d'autres groupes de femmes ont été a l'origine de ces changements, on peut toujours se demander si effectivement l'Église ne réagit pas à une demande sociale, comme en Belgique.

Une autre interrogation porte sur l'importance des cercles comme lieu d'autonomisation des fermières; cette hypothèse vérifiée pour le Québec, l'est-elle pour la Belgique? L'état actuel des recherches sur le sujet dans ce pays n'apporte pas, pour le moment, de réponse satisfaisante à une question qui dépasse largement le cadre de cette étude. Cependant, la question des origines de classe et de l'interprétation que l'on fait de la vie publique entrent en ligne de compte. Ainsi, il est clair que les fermières, membres des cercles, d'abord recrutées au sein des élites locales, s'affirment comme des «personnalités» de leur paroisse; elles ont accédé à la vie publique par le biais de leur association, affirme Yolande Cohen. Pour les châtelaines et dames nobles, 
qui en Belgique assumaient dès l'origine les plus hautes fonctions, parfois honorifiques, que signifie cet accès à la vie publique? Ne l'avaient-elles pas grâce à leur statut? La comtesse de Villermont, pionnière du mouvement, ou la baronne Rotsart de Hertaing, présidente du Comité national (sur lesquelles, par ailleurs, il serait bon d'effectuer des recherches) prennent, nous semble-t-il, une place nouvelle dans la vie publique grâce à des fonctions effectives au sein des comités provinciaux et nationaux des cercles. Si certaines autres femmes avaient déjà eu l'occasion d'exercer des responsabilités dans le cadre d'associations charitables, comme la Ligue des Femmes Chrétiennes créée par le révérend père Van Langermeersch en 1892, ces dernières jouent un rôle non négligeable de représentation internationale et nationale ${ }^{57}$. Ce rôle est nouveau parce qu'il se démarque, de par les objectifs poursuivis, de l'œuvre de bienfaisance. Enfin, d'autres femmes, tant au Québec qu'en Belgique, ont effectué des tâches à l'intérieur des associations de fermières qui les ont amenées elles aussi à affronter la vie publique: songeons aux présidentes et aux secrétaires des cercles, mais également aux institutrices des écoles ménagères qui ont contribué à l'élaboration d'un savoir dont, en plus, elles ont été les principaux vecteurs.

Nous avons voulu aussi soulever d'autres points qui ont suscité la controverse à la lumière d'éléments comparatifs. L'histoire des cercles belges reste à faire et ne peut donc ici être analysée en profondeur. Toutefois, l'analyse de leur moment de fondation en oriente le déroulement: au contraire des cercles au Québec, les cercles belges périclitent après avoir été très dynamiques. On ne peut s'empêcher de penser que leur relation organique à l'Église les a contraints à réduire leur champ d'intervention aux seuls domaines autorisés par elle et par conséquent à manquer de l'autonomie qui fut si salutaire aux cercles du Québec pour recruter et pour déployer leur organisation ${ }^{58}$.

L'autre interrogation majeure que nous voudrions soulever au terme de cette analyse relève d'une préoccupation politique. Même si nous n'avons pas présenté l'ensemble des données concernant les partis politiques au pouvoir au cours de ces années, le problème du corporatisme avoué de ces associations doit être abordé. Cette idéologie prend un visage nouveau dans les années 1930 avec les résultats

57. L'initiative féminine, revue officielle illustrée de toutes les œuvres de femmes, direction et administration: secrétariat général des œuvres féminines, Bruxelles, 1912-1914, 1 (mai 1913).

58. M. Pastur, membre de la chambre des représentants en Belgique, regrette le peu de place laissé à l'économie dans les cercles belges. Il propose la création d'associations d'achats et de ventes en commun, intervention dans III ${ }^{\text {eme }}$ Congrès international des Cercles de fermières de Gand, 1913, Bruxelles, volume 4, Procès verbaux de la troisième section. 
que l'on sait dans des pays comme l'Allemagne nazie et l'Italie fasciste. Certes, l'idéologie corporatiste prônée par l'Église n'est pas du même ordre, encore qu'en Italie elle ait favorisé son rapprochement avec Mussolini et la signature des accords du Latran. On peut se demander si en érigeant l'image d'une société sans classes, où la complémentarité des rôles sexuels établie sur l'assujettissement de la femme à l'homme serait la clé de voûte de cet édifice, l'idéologie cléricale n'a pas contribué à maintenir et à renforcer la séparation des sphères masculines et féminines dans l'ignorance de leur condition réelle. Cette critique, connue, n'épuise toutefois pas la question. On se hasardera sur une autre piste; on se demandera au contraire si en structurant le milieu rural, et en ne s'opposant pas à ce que les femmes se dotent d'organisations solidement constituées, l'Église, et accessoirement l'État, n'ont pas d'une certaine manière préconisé une forme idéale de corporatisme qui aurait ralenti le déferlement du corporatisme fasciste en milieu rural. Même s'il y avait de nombreux partisans fascistes dans les deux pays, leur victoire demeurait toutefois peu probable compte tenu de tous les autres facteurs en jeu. Néanmoins, en avançant cette hypothèse, on ouvre le débat en appelant de nos vœux son approfondissement qui passe peut-être aussi par une comparaison plus large avec les femmes italiennes, dont l'histoire est racontée par V. De Grazia et M. de Giorgio ou les femmes allemandes, auxquelles $\mathrm{G}$. Bock a su si bien nous intéresser. 